\title{
Prognostic Significance of B Type Natriuretic Peptide in Acute Coronary Syndrome
}

Nasreen Chowdhury ${ }^{1 *}$ Md. Aminul Haque Khan ${ }^{2}$ Md Mozammel Hoque ${ }^{3}$

'Department of Biochemistry Chattagram Maa-O-Shishu Hospital Medical College Chittagong, Bangladesh

${ }^{2}$ Department of Biochemistry Enam Medical College, Savar Dhaka, Bangladesh.

${ }^{3}$ Department of Biochemistry

Bangabandhu Sheikh Mujib Medical University Dhaka, Bangladesh.

${ }^{*}$ Correspondence to:

Professor Nasreen Chowdhury

Department of Biochemistry

Chattagram Maa-O-Shishu Hospital Medical College Chittagong, Bangladesh.

Mobile: +8801711331160

E-mail:drnasreenchy@yahoo.com
Acute Coronary syndrome (ACS) is the most common cause of admission to the coronary care unit with highest risk of death and adverse outcomes. ACS accounts for $60-70 \%$ of all admissions in the hospital. Patients with ACS encompass a heterogeneous group that varies widely regarding severity of the underlying coronary artery disease, prognosis and response to treatment. Patients with the highest risk of subsequent events usually have the largest benefit of an intensified pharmacological treatment and early mechanical intervention. The prognosis for low-risk patients, on the other hand, is often difficult to improve further and these patients usually benefit more from a conservative management with a lower risk of side effects. Therefore, risk stratification is essential and should be initiated early and updated continuously throughout the hospital stay.

Early risk stratification is usually performed by the use of clinical background factors, clinical presentation, electrocardiography and biochemical markers of myocardial damage. Levels of natriuretic peptides have been shown to reflect cardiac performance. The aim of this study was to review elaborately on B type Natriuretic Peptide (BNP) and its prognostic value in patient with ACS. This review focuses on the emerging role of these peptides in the early risk stratification of ACS patients. Elevation of BNP levels in acute MI and UA is predictive of a greater risk of death, post infarction heart failure, or reinfarction. Post infarction studies demonstrate that elevated plasma BNP levels are associated with larger infarct size, increased probability of ventricular remodeling, lower ejection fraction, higher risk of heart failure, and increased mortality. This cardiac marker is a potent predictor of mortality in patients with all forms ACS. BNP measurements serve as an index of severity of the ischemic injury, as well as the degree of impairment in left ventricular function.

Key words: Acute coronary syndrome (ACS); B-type natriuretic peptide (BNP); risk stratification.

\section{INTRODUCTION}

The acute coronary syndrome (ACS) includes acute myocardial infarction (AMI) with ST segment elevation or depression and unstable angina (UA). This syndrome is a serious health problem because it is responsible for $20 \%$ of all medical emergency admission and the highest risk of disease events and death ${ }^{1}$. Unstable angina and nonST segment elevation myocardial infarction account for about 2.5 million hospital admissions worldwide and are major causes of mortality and morbidity. The prognosis is substantially worse than for chronic stable angina and affect $5-10 \%$ of hospital death and re-infarction. Despite optimal treatment by anti-ischemic and antithrombotic drugs, death and recurrent myocardial infarction occur in another 5$10 \%$ of patients within the month after an acute episode. Several studies indicate that these patients may have a higher long term risk of death and myocardial infarction than do patients with ST segment elevation ${ }^{2}$. 
Hyperlipidemia is a critical factor in $50 \%$ of patients with atherosclerotic cardiovascular disease; however, in recent years additional inciting factors (inflammatory and neurohormonal) have been identified. Chronic vascular inflammation is a dynamic process of the arterial vessel wall where cycles of inflammatory and thrombotic activity play a critical role in the development of atherothrombosis, resulting in acute coronary syndrome ${ }^{3}$.

Atherosclerotic lesion represents a cascade of cellular and molecular assaults on vascular endothelium beginning early in life. Factors such as elevated level of low density lipoprotein cholesterol, cigarette smoking, elevated homocystiene levels, infectious agents and genetic predisposition inflict injury on endothelium, triggering compensatory responses that alter normal endothelial function and environment. As a whole the injurious process triggers a chronic and complex inflammatory process that involves migration of destructive substances, cycles of cellular proliferation and necrosis and remodeling of lesion. Ultimately the core of lipid and necrotic tissue becomes covered by a fibrous cap. Neurohormonal and inflammatory factors contribute to the destabilization and rupture of susceptible atherosclerotic plaques, leading to coronary thrombosis and acute coronary events ${ }^{3}$.

The usual approach to the treatment of an acute coronary event involves early aggressive therapeutic strategies aimed at limiting the amount of myocardial injury and preventing complications. In order to target high risk population for therapy, the challenge has been to identify those patients. Despite the advent of chest pain center and sensitive biomarkers of myocardial necrosis, a number of patients presenting with chest pain to the emergency department after being evaluated and released on the basis of normal biomarkers subsequently return with an acute MI within 48 hours ${ }^{4}$.

The primary challenge is the early and specific diagnosis of acute coronary events that helps to initiate appropriate therapy without delay. Recently, The American College of Cardiology and American Heart Association Guidelines have recommended that risk stratification is one of the most important initial steps in evaluation and treatment of disease ${ }^{5}$.

Traditional serum markers of myocardial injury in acute coronary events (cardiac enzymes, troponin and myoglobin) reflect only the sequel of inflammatory milieu and plaque rupture. In contrast to the injury markers, newly identified serum substances have drawn attention for their ability to portend acute clinical events and their outcomes. Most markers of myocardial cell injury that reflect structural consequences of coronary ischemia [cardiac enzyme, cardiac troponin (cTn)] do not increase until 3 to 4 hours after the onset of pain. The clinical evaluation of ECG findings and the traditional marker provide only partial information of risk stratification. New biomarkers like natriuretic peptide may provide additional pathophysiological insight and add to a strategy for comprehensive assessment ${ }^{6}$.
B type natriuretic peptide (BNP) is a 32 amino acid peptide released from ventricular myocardium predominantly in response to increased ventricular wall stress. It is synthesized as a prohormone that is cleaved at $\mathrm{N}$ terminal to produce BNP and NT-proBNP. Along with ANP (atrial natriuretic peptide), BNP has effects on natriuresis, diuresis and inhibition of reninangiotensin-aldosterone system all of which contribute to the modulation and control of cardiovascular hemodynamics ${ }^{7}$.

Both BNP and NT-proBNP have been shown to aid in the diagnosis of heart failure and to correlate with functional status among patients with congestive heart failure ${ }^{8}$. There is a robust association between BNP and the short and long term risk of death across the spectrum of non-ST elevation ACS including patients without myocardial necrosis or clinical evidence of heart failure 6, 9-12. $^{2}$.

ACS with an elevated BNP level may be blessings in disguise since it heralds the point of early invasive management strategy as has also been demonstrated for troponin $\mathrm{T}$ and I. In patient with ACS, BNP adds important prognostic information to clinical and laboratory variables including ECG, echo, troponin and CRP. The magnitude of risk relationship associated with BNP seems to be greater than that associated with most currently available markers. Moreover, investigations (e.g. echo) most commonly used to assess prognosis is not available in many health care systems where BNP can be a potential diagnostic and prognostic aid. This simple, relatively inexpensive and rapid test improves the ability of clinicians for risk assessment among patients with ACS who are at high mortality risk. BNP provides a convenient and noninvasive means to gain insight into the underlying consequences of ACS that mediate the risk of recurrent events and thereby may identify target causes for specific therapeutic intervention.

Since BNP has the potential to improve substantially the outcome in patients with ACS by facilitating its risk assessment and timely clinical decision making process, we have decided to review elaborately on BNP and its prognostic value in patients with ACS and to review its potentials in comparison to other markers for risk assessment in patients with ACS.

\section{BIOMARKERS IN ACS}

Recent studies have examined the role of nontraditional biomarkers in the risk stratification of patients with ACS. Highsensitivity C-reactive protein (hs-CRP), a marker of inflammation, has been shown to provide prognostic information in patients with ACS, independent of clinical factors and traditional markers of necrosis ${ }^{13-15}$. B-type natriuretic peptide (BNP) has been associated with heart failure, as well as adverse clinical outcomes (predominantly mortality), in patients with $\mathrm{ACS}^{16}$. The study findings suggest that future risk stratification in patients with acute coronary syndrome may involve a panel of biomarkers ${ }^{13-16}$. One investigative team has proposed a simplified method of combining the information provided by biomarkers ${ }^{17}$. 
From zero to three points are assigned, depending on the number of elevated biomarkers (cardiac-specific troponin, hsCRP, BNP). The risk of death, recurrent myocardial infarction, or congestive heart failure has been found to be 4.5 times higher when all three biomarkers are elevated than when no biomarker is elevated. However, more data are needed before use of hs$\mathrm{CRP}$ and BNP can be recommended for risk stratification in UA/NSTEMI.

Role of biochemical markers in acute coronary syndrome Contribution of Laboratory Medicine to clinical cardiology has grown fast over the years. During the later part of the $20^{\text {th }}$ century, highly sensitive and specific cardiac markers have been introduced for the detection of myocardial damage, (e.g. cardiac troponins), as well as for the assessment of myocardial function, (e.g. cardiac natriuretic peptides).

Outcomes in ACS are influenced by the speed and accuracy of diagnosis and the timely appropriate therapy. Most traditional markers of myocardial cell injury secondary to coronary ischemia do not increase until 3 to 4 hours after the onset of pain. Novel markers of plaque inflammation and neurohormonal activation make early diagnosis of ACS possible. That helps determine the need for aggressive therapeutic interventions.

\section{Novel markers of acute coronary syndrome}

These are pregnancy associated plasma protein-A (PAPP-A), highly sensitive cross reactive protein (hs-CRP), plasma DDimer, interleukin-6 (IL-6), myploperoxidase (MPO), troponins and cardiac natriuretic peptides.

\section{Cardiac natriuretic peptides}

Cardiac natriuretic peptides include atrial natriuretic peptide (ANP) and B-type natriuretic peptide (BNP), while other natriuretic peptides, such as C-type natriuretic peptide and urodilatin, are not produced and secreted by cardiac tissue but by other tissues ${ }^{18}$. ANP and BNP derive from precursors, the pre-pro-hormones, which contain a signal peptide sequence at the N-terminal end ${ }^{19}$. The pro-hormones are further split into inactive N-terminal fragments and the biologically active peptide hormones ${ }^{19}$. ANP is secreted mainly from atrial cardiomyocytes whereas BNP is preferentially produced and secreted from the left ventricle, although right side of heart can also synthesize and secrete BNP in response to disease ${ }^{20}$. The precise mechanisms controlling production and secretion of cardiac natriuretic peptides are still unclear, although ventricular stretch and wall tension are likely to be important. In general, the plasma concentrations of these peptides are increased in diseases characterized by volume expansion (e.g renal failure, primary hyper aldosteronism congestive heart failure ctc, or by ventricular hypertrophy or strain, (e.g thyroid disease, excessive circulating glucocorticoid or hypoxia $)^{19}$.
Although the reliable role of cardiac natriuretic peptides in the identification and management of patients with symptomatic and asymptomatic ventricular dysfunction remains to be fully clarified, the clinical usefulness of cardiac natriuretic peptides (especially BNP and NTpro-BNP) in the evaluation of patients with suspected heart failure, in prognostic stratification of patients with $\mathrm{CHF}$, in detecting LV systolic or diastolic dysfunction and in the differential diagnosis of dyspnea has been confirmed even more recently ${ }^{21}$. BNP and NTpro-BNP have also emerged as prognostic indicators of long-term mortality early after an acute coronary event. This association was observed across the spectrum of ACS, irrespective of serum concentration of troponin and clinical evidence of heart failure. Natriuretic peptide concentrations were also found related to risk of cardiovascular events and death in apparently asymptomatic persons ${ }^{22}$.

\section{B-type natriuretic peptide}

\section{Structure}

The circulating (biological active) form of human BNP, as shown in the figure, comprises a 32 amino acid peptide with a 17 amino acid ring closed by a disulfide bond between two cysteine residues. Its amino acid sequence is highly conserved across species.

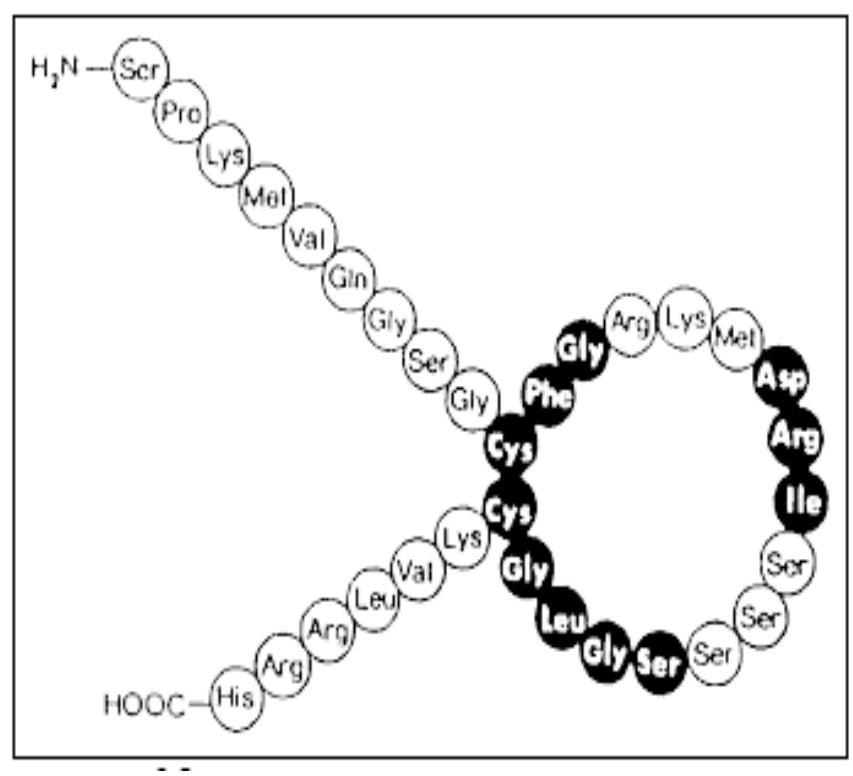

Figure 1 : BNP 32 (the biologically active hormone)

The first natriuretic peptide ever discovered was atrial natriuretic peptide (ANP), BNP is secreted by myocardial cells located on both atria and ventricles, The name was given because it was first discovered in porcine brain. 
Biosynthesis of B-type natriuretic peptide

BNP's gene is located on the short arm of chromosome 1, close to ANP loci. Transcription yields a precursor containing a hydrophobic leader sequence that is translated to a chain of 108 amino acids (proBNP) that coexist with ANP in some of the secretory vesicles of the atrial and ventricular myocardial cells $\mathrm{s}^{23-24}$. The biologically active peptide sequence lies in the carboxyl-terminal portion of the pro-hormone and the cleavage of the proBNP occurs between the Arg and Ser amino acid residues $^{25}$. The processing site in proBNP occurs immediately after the Arg-X-X-Arg sequence, resulting in mature BNP molecules varying in length from 26 to 45 amino acids. Processing of the precursor releases a mature BNP (32 amino acid) and an amino-terminal fragment (NTproBNP), an endocrinologically inactive molecule, both of which circulate in the plasma ${ }^{26-28}$.

\section{BNP use in clinical practice}

\section{Diagnosis of heart failure}

Not have heart failure is quite useful (McGeoch et al., 2002, McCullough et al., 2002).

Plasma concentration of BNP increases in heart failure, especially in highly symptomatic patients. It has been proposed that patients with BNP plasma concentrations of $<20 \mathrm{pmol} / \mathrm{L}$ have very few probabilities of suffering from heart failure, while patients with higher concentrations must be submitted to further investigations of their cardiovascular system. Recent guidelines for the diagnosis of heart failure have included this proposal. Diastolic dysfunction and valvular disorders both can lead to the increase of BNP concentration ${ }^{29}$. So patients with increased BNP levels must undergo further investigations, in order to estimate the etiology and pathophysiology of the underlying disease.

\section{Left ventricular dysfunction diagnosis}

In patients with normal systolic function verified ultrasonografically, increased BNP levels correlate well with Doppler measurements confirming diastolic dysfunction of the left ventricle. Patients with asymptomatic dysfunction of the left ventricle are expected to have lower BNP plasma concentrations than patients with severe heart failure. The more severe the dysfunction of the left ventricle, the higher the BNP plasma concentration $^{30-31}$.
Diagnosis of left ventricular systolic dysfunction after AMI

BNP plasma concentration increases after acute myocardial infarction and this increase correlates to the severity of the infarction. Patients with smaller infarcts show an increase of BNP 20 hours after the initiation of symptoms. Patients with larger infracts, lower ejection fraction and more frequent signs of heart failure, reach maximum BNP levels 5 days after admission. In the acute phase, BNP values do not reflect patient's hemodynamic profile, but four days later BNP levels correlate well with the ejection fraction of the left ventricle and with pulmonary wedge pressure ${ }^{11,17,32}$.

\section{Diagnosis of Right ventricular failure}

Patients with pulmonary hypertension have increased BNP levels because of its increased production in the right ventricle. The level of the increase correlates well with the level of dysfunction of the right ventricle in patients with pulmonary hypertension or congenital heart failure.

\section{Prognosis}

\section{Heart failure prognosis}

Until today, published studies indicate that BNP (or NTproBNP) concentration provides important prognostic information in heart failure patients and support its use as an adjuvant to clinical assessment, especially at centers where risk stratification is of great importance (i.e. transplantation units). Further research is necessary to clarify whether BNP plasma levels are a satisfactory means of identifying patients with low risk for future hospitalizations $\mathrm{s}^{30-31}$.

\section{Prognosis after acute coronary syndrome}

Increased BNP levels on admission in patients that have had an acute coronary syndrome without ST elevation, increases the possibility of both short-term and long-term complications. Recent studies indicate that the prognostic value of NTproBNP assessment in patients with coronary syndrome is very high and that NT-proBNP is an independent predictive factor of the severity of the coronary artery disease. Patients with NT-proBNP $>1654$ $\mathrm{ng} / \mathrm{L}$ had 27 times greater risk of dying than patients with NT-proBNP $<122 \mathrm{ng} / \mathrm{L}^{16,30,33-34}$

\section{Prognosis in the general population}

In all studies, BNP plasma concentration represents an independent predictive factor for survival, irrespective of the existence of coronary artery disease. Assessing NTproBNP concentration didn't seem to add further information ${ }^{35}$. 


\section{Prognosis in pulmonary hypertension}

In pulmonary hypertension patients, prognosis is worse for those with higher BNP plasma levels, a 4-fold increase of mortality within 2 years has been noted in patients with BNP values twice above the normal ${ }^{36-37}$.

\section{Therapy assessment}

There are indications that assessing BNP levels and adjusting therapy (dosage increase) according to $\mathrm{it}^{18}$. Intravenous administration of synthetic human BNP (nesiritide) in heart failure patients causes dilation of the arteries and veins without causing any change to the heart rhythm, thus leading to increased cardiac output due to increased stroke volume. It also promotes diuresis, either because of the increased cardiac output or because of a direct natriuretic action. Studies have been conducted using the peptide for up to seven days with no allergic reactions noted. The most important side effect is hypotension, which is, nonetheless, dose-related. In July 2001, the FDA approved the administration of BNP in the treatment of heart failure.

\section{Assessing BNP and NT-proBNP concentration in plasma}

The first method used to assess the plasma concentration of natriuretic peptides was radio-immunological (RIA), but its application was impractical, as it was time-consuming, complex and required a relatively large quantity of blood. To overcome these problems, researchers introduced a new immunoradiometric method (IRMA) that used two specific monoclonal antibodies against two loci of the BNP chain. BNP plasma concentration increases with age and is slightly higher in women. The proposed normal range of values is $0.5-3.0 \mathrm{pg} / \mathrm{mL}$ (0.15-0.90 pmol/L), for both RIA and IMRA. The proposed threshold for the diagnosis of heart failure in patients over 55 years old, referring to the method commercially available (Biosite Diagnostics), is $80 \mathrm{pg} / \mathrm{mL}$. For NT-proBNP, proposed normal range of values is $68-112 \mathrm{pg} / \mathrm{mL}(8.2-13.3 \mathrm{pmol} / \mathrm{L})$.

\section{SOME STUDIES ON BRAIN NATRIURETIC PEPTIDE AS A PROGNOSTIC MARKER IN ACUTE CORONARY SYNDROME}

The use of natriuretic peptides (especially BNP and NTproBNP) is well-established in the diagnosis and staging of patient with heart failure. Its ability to predict prognosis in population has been verified in several frequently cited studies.

In 1994, Arakawa et al. recognized the positive relationship between plasma level of BNP and myocardial infarct size ${ }^{38}$. In 1996, the researchers had gone through another study to seek out whether plasma BNP concentration can predict prognosis after MI. BNP and ANP levels were measured in 70 patients with AMI. Measurements were obtained on admission and on day 2 following MI. Mean follow-up period was 18 months.
Both values of plasma BNP correlated significantly with hemodynamic variables which were influenced by left ventricular function. By Kaplan Meier analysis it was found that patient with plasma BNP levels higher than median levels on admission and day 2 had significantly higher mortality rates than those with the sub-median level. The researcher concluded that plasma BNP concentration in early and sub-acute phase of MI was a powerful noninvasive indicator of poor prognosis, possibly reflecting residual left ventricular function after $\mathrm{MI}^{39}$. In another study, Omland et al. found the diagnostic and prognostic value of plasma BNP as an indicator of left ventricular dysfunction and long-term survival after AMI, relative to that of ANP and N-ANP ${ }^{40}$. Venous blood samples for analysis of ANP, N-ANP, and BNP were obtained on day 3 after symptom onset from 131 patients with AMI. Left ventricular ejection fraction was determined by echocardiography in a subsample of 79 patients. Twenty-eight cardiovascular and 3 noncardiovascular deaths occurred during the follow-up period (median, 1293 days). All three peptides proved to be powerful predictors of cardiovascular mortality by univariate Cox proportional hazards regression analyses. In a multivariate model, plasma BNP but not ANP or N-ANP provided additional prognostic information beyond left ventricular ejection fraction. Logistic regression analysis showed that ANP and N-ANP but not BNP were significantly associated with a left ventricular ejection fraction $45 \%$. These results suggest that plasma BNP determination provides important, independent prognostic information after AMI. Although plasma ANP appears to be a better predictor of left ventricular dysfunction, plasma BNP may have greater potential to complement standard prognostic indicators used in risk stratification after AMI because of its strong, independent association with long-term survival, enhanced in vitro stability, and simplicity of analysis.

Although the circulating level of this neurohormone has been shown to provide independent prognostic information in patients with MI, few data are available for patients with ACS in the absence of ST-segment elevation. A study was carried out by de Lemos et $\mathrm{al}^{16}$. (2001) where, B-type natriuretic peptide in plasma specimens was obtained a mean $( \pm$ SD) of $40 \pm 20$ hours after the onset of ischemic symptoms in 2525 patients with UA/NSTEMI. Results show the base-line level of B-type natriuretic peptide was correlated with the risk of death, heart failure, and myocardial infarction at 30 days and 10 months. The unadjusted rate of death increased in a stepwise fashion among patients in increasing quartiles of base-line BNP levels. This association remained significant in subgroups of patients with STEMI, NSTEMI and UA. After adjustment for independent predictors of the long-term risk of death, the odds 
ratios for death at 10 months in the second, third, and fourth quartiles of B-type natriuretic peptide were 3.8, 4.0 and 5.8. The level of B-type natriuretic peptide was also associated with the risk of new or recurrent MI and new or worsening heart failure at 10 months.

A study was done by Jernbergh et al. where the NT-proBNP was analyzed on admission in 755 patients with chest pain and no ST-segment elevation ${ }^{11}$. Patients were followed concerning death for 40 months (median).The median NT-proBNP level was $400 \mathrm{ng} / \mathrm{L}$. Compared to the lowest quartile, patients in the second, third and fourth quartiles had a relative risk of subsequent death of 4.2, 10.7 and 26.6 respectively. When NTproBNP was added to a Cox regression model including clinical background factors, ECG and troponin T, the NT-proBNP levels were independently associated with prognosis.

It has been proved that, BNP is a predictor of short- and medium-term prognosis across the spectrum of ACS. NTproBNP may be an even stronger prognostic marker. To assess whether NTproBNP is a powerful prognostic marker of long-term, all-cause mortality Omland et $\mathrm{al}^{9}$. conducted another study in a large, contemporary cohort of patients with ACS. Blood samples for NTproBNP determination were obtained in the subacute phase in 204 patients with STEMI, 220 with NSETMI and 185 with UA. After a median follow-up of 51 months, 86 patients (14\%) had died. Median NTproBNP levels were significantly lower in long-term survivors than in patients dying (442 versus $1306 \mathrm{pmol} / \mathrm{L}$ ). In a multivariate Cox regression model, NTproBNP (risk ratio 2.1) added prognostic information above and beyond Killip class, patient age, and left ventricular ejection fraction. Adjustment for peak troponin $\mathrm{T}$ levels did not markedly alter the relation between N-BNP and mortality. In patients with no evidence of clinical heart failure, N-BNP remained a significant predictor of mortality after adjustment for age and ejection fraction. Finding concludes that $\mathrm{N}-\mathrm{BNP}$ is a powerful indicator of long-term mortality in patients with ACS and provides prognostic information above and beyond conventional risk markers.

The utility of using biomarkers in combination was also studied by Sabatine et $\mathrm{al}^{17}$. Baseline measurements of TnI, CRP, and BNP were performed in 450 patients in OPUS-TIMI 16. Elevations in each TnI, CRP, and BNP were independent predictors of the composite of death, myocardial infarction (MI), or congestive heart failure (CHF). The same study group also designed another study to evaluate B-type natriuretic peptide (BNP) for risk assessment and clinical decision making over a range of cut points, alone and with cardiac troponin I (cTnI), in patients with non-ST-elevation ACS.

Morrow et al. evaluated BNP at baseline in 1,676 patients with non-ST-elevation ACS randomized to early invasive versus conservative management ${ }^{12}$. Patients with elevated BNP $(>80$ $\mathrm{pg} / \mathrm{mL} ; \mathrm{N}=320$ ) were at higher risk of death at seven days and six months. The association between BNP and mortality at six months was independent of important clinical predictors, including cTnI and congestive heart failure (CHF). Patients with elevated BNP had a five-fold higher risk of developing new $\mathrm{CHF}$ by 30 days. B-type natriuretic peptide added prognostic information to cTnI, discriminating patients at higher mortality risk among those with negative and positive baseline cTnI results. No difference was observed in the effect of invasive versus conservative management when stratified by baseline levels of BNP. They concluded that elevated BNP $(>80 \mathrm{pg} / \mathrm{mL})$ at presentation identifies patients with non-ST-elevation ACS who are at higher risk of death and CHF and adds incremental information to cTnI.

In a combination marker study by James et al., the associations between $\mathrm{N}$-terminal pro-brain natriuretic peptide (NT-proBNP) and other biochemical and clinical risk indicators, as well as their prognostic value concerning the individual end points of death and myocardial infarction (MI), were elucidated in a large cohort of ACS patients ${ }^{6}$. NTproBNP, troponin $\mathrm{T}$, and $\mathrm{C}$-reactive protein (CRP) were analyzed in blood samples obtained at a median of 9.5 hours from symptom onset in 6809 of 7800 ACS patients in the Global Utilization of Strategies To Open occluded arteries-IV (GUSTO-IV) trial. Levels of NT-proBNP correlated independently with age, female gender, low body weight, diabetes, renal dysfunction, history of MI, heart failure, heart rate, ongoing myocardial damage, and time since onset of ischemia. Increasing quartiles of NT-proBNP were related to short- and long-term mortality that reached $1.8 \%, 3.9 \%$, $7.7 \%$, and $19.2 \%$ respectively, at 1 year. Levels of troponin T, CRP, heart rate, and creatinine clearance, in addition to STsegment depression, also correlated independently with 1year mortality, but NT-proBNP was the marker with the strongest relation. They concluded that the use of NTproBNP appears to add critical prognostic insight to the assessment of patients with ACS.

In a separate study, Saribulbul et al. prospectively examined brain natriuretic peptide levels in the plasma of 26 patients undergoing coronary artery bypass grafting ${ }^{41}$. Brain natriuretic peptide measurements were carried out 4 times preoperatively, 3 hours after institution of cross-clamping, 24 hours after institution of cross-clamping, and on the $5^{\text {th }}$ postoperative day. In addition, they measured individual variables and compared them to brain natriuretic peptide levels. Mean preoperative brain natriuretic peptide levels were significantly higher in patients with histories of myocardial infarction and heart failure (ejection fraction 0.40). There was a significant correlation between preoperative brain natriuretic peptide levels and cross-clamp times and an inverse correlation between those levels and preoperative cardiac indices. The preoperative brain natriuretic peptide level also correlated inversely with left ventricular ejection fraction before and 5 days after operation. When the Clinical Severity Scoring System was applied, preoperative brain natriuretic peptide plasma concentrations in 19 patients with risk scores of 0-2 were significantly lower than in the 7 patients whose risk scores were $3-6$. There was also a significant relationship between preoperative brain natriuretic peptide plasma concentrations and the postoperative requirement for inotropic agents. This study suggests that plasma BNP concentration could be one of the predictors of risk in patients undergoing coronary artery bypass grafting. 
The role of N-terminal pro-brain natriuretic peptide (NTproBNP) in the early treatment of patients with non-STelevation acute coronary syndrome (NSTACS) has been evaluated again by Jernbergh et al. in 6 large studies that include $>12,000$ patients $^{42}$. These studies convincingly show that this marker of cardiac performance is associated strongly with death and the risk of future congestive heart failure and carry important prognostic information that is independent from previous known risk factors in NSTACS.

Bazzino et al., prospectively studied the additive value of $\mathrm{N}$ terminal probrain natriuretic peptide (NT-proBNP) in relation to the Thrombolysis in Myocardial Infarction (TIMI) risk score and the American College of Cardiology/American Heart Association (ACC/AHA) joint prognostic classification, and compared the predictive capacity of NT-proBNP, troponin T (TnT), C-reactive protein (hsCRP), myoglobin, and creatine kinase-MB (CK-MB) concentrations in a cohort of 1483 consecutive patients with non-ST-segment-elevation acute coronary syndromes (NSTE-ACS ${ }^{43}$. Centralized measurements of NT-proBNP, TnT, myoglobin, and hsCRP were performed 3 $\mathrm{h}$ (median) after admission. Adjusting by clinical, ECG variables, and biomarkers, NT-proBNP concentration was the strongest independent predictor of in-hospital (OR 1.7) and 180-day mortality (OR 1.67) and added significant prognostic information to the TIMI and ACC/AHA prognostic categories.

In a study by Yang et al., value of circulating BNP was evaluated for early percutaneous coronary intervention (PCI) in patients with ACS. Nine hundred and sixty consecutive patients with ACS were enrolled ${ }^{44}$. Circulating BNP level was measured when each patient arrived at the emergency room. All patients underwent PCI in 90 minutes in spite of contraindication. Cardiac events (death from any cause, heart failure, and recurrence of acute myocardial infarction or ACS) were recorded during follow-up. In patients with BNP $80 \mathrm{pg} / \mathrm{mL}$, mortality from all causes within 1 month and 6 months in those underwent delayed PCI ( 6 hours) was significantly higher than those received early PCI ( $<6$ hours). Similarly, the incidence rate of heart failure in delayed PCI patients was significantly higher than those received early PCI within 1 month and 6 months. The recurrence rate of AMI or ACS was not significantly different between early PCI and delayed PCI patients with BNP level $80 \mathrm{pg} / \mathrm{mL}$.

Galvani et al. reported a study where N-terminal pro-brain natriuretic peptide (NT-proBNP) levels were measured on admission in patients with ACS and ECG evidence of myocardial ischemia ${ }^{45}$. The NT-proBNP was measured at a median time of 3 hours after symptom onset in 1756 patients. The outcome measure was death at 30 days, which occurred in 113 patients $(6.4 \%)$. The median NT-proBNP level was 353 ng/L. Compared with the lowest quartile, patients in the second, third, and fourth quartiles had a relative risk of subsequent death of 2.94, 5.32, and 11.5 respectively. The NT-proBNP was independently associated with death in a logistic regression model, which included clinical variables, ECG, and troponin $\mathrm{T}$ in patients either with or without persistent ST-segment elevation. NT-proBNP was also an independent predictor of severe heart failure. They concluded that the measurement of NT-proBNP on admission improves the early risk stratification of patients with ACS, suggesting the need for the development of targeted therapeutic strategies.

Ollivier et al. measured troponin and NT-pro BNP plasma concentrations on emergency admission and 8 and 24 hours later in 64 patients with $\mathrm{ACS}^{46}$. Troponin levels were abnormal in respectively $44 \%, 51 \%$ and $52 \%$ of patients, while NT-pro BNP levels were abnormal in $75 \%, 83 \%$ and $79 \%$ of patients. Both troponin and NT-pro BNP levels were abnormal in patients with ST elevation MI and in patients with non ST elevation MI. In contrast, among 30 patients with unstable angina, troponin levels were always normal whereas NT-pro BNP levels were elevated in $73 \%$ of cases.

Suzuki et al. studied whether the plasma BNP level measured 3 to 4 weeks after the onset of AMI represents a reliable prognostic marker for patients with $\mathrm{AMI}^{47}$. They analyzed 145 consecutive patients with AMI. Plasma BNP levels were measured during the 3 to 4 weeks after onset of AMI. Of those patients, 23 experienced fatal cardiac events during this study. The mean follow-up period was 58.6 months. Log BNP, left ventricular end-diastolic pressure, and pulmonary vascular resistance were all significantly higher in the cardiac death group.

Jarai et al. had done another study to compare the accuracy of the NTproBNP and NT-proANP in the prediction of the 2-year mortality and to investigate whether additional measurement of NT-proANP to troponin I (TnI) could improve risk assessment in the subgroups of patients with unstable coronary artery disease (UCAD) and normal NT-proBNP ${ }^{48}$. Plasma levels of the TnI, NT-proANP, and NT-proBNP were determined in 120 consecutive patients with UCAD without ST-segment elevations and normal left ventricular function. In multivariable logistic regression analysis, TnI and NT-proBNP were independent predictors of mortality.

In a prospective study, Wang et al. examined the relations of plasma BNP and NTproBNP to the risk of death from any cause like heart failure, atrial fibrillation, stroke or transient ischemic attack and coronary heart disease ${ }^{22}$. During a mean follow-up of 5.2 years, 119 participants died and 79 had a first cardiovascular event. After adjustment for cardiovascular risk factors, each increment of $1 \mathrm{SD}$ in $\log$ BNP levels was associated with a $27 \%$ increase in the risk of death, a $28 \%$ increase in the risk of a first cardiovascular event, a $77 \%$ increase in the risk of heart failure, a $66 \%$ increase in the risk of atrial fibrillation, and a $53 \%$ increase in the risk of stroke or transient ischemic attack.

Elevated baseline levels of BNP and NTproBNP have been associated with adverse long-term outcome in patients with ACS, whereas the prognostic implications of serial NT-proBNP measurements had not been investigated. NT-proBNP, troponin $\mathrm{T}$, and C-reactive protein were measured by Heeschen et al. at baseline and at 48 and 72 hours in 1791 patients with non-STelevation $\mathrm{ACS}^{49}$. Death and MI were recorded during 30 days of follow-up. After adjustment for independent predictors of cardiac risk, baseline NT-proBNP levels $>250 \mathrm{ng} / \mathrm{L}$ were associated with higher event rates. 
Kirstop et al. had done a prospective study to assess the prognostic value of the NT-proBNP vs. CRP and urinary albumin/creatinine ratio in an older adult population ${ }^{50}$. Among 764 participants (aged 50 to 89 years) 658 participants provided blood and urine samples which were examined. Of these participants, 626 without heart or renal failure were enrolled. A subgroup of 537 had no history of cardiovascular disease at baseline. During 5 years of follow-up 94 participants died and 65 developed a first major cardiovascular event. Researchers commented that measurements of NT-proBNP provide prognostic information of mortality and first major cardiovascular events beyond traditional risk factors. NTproBNP was a stronger risk biomarker for cardiovascular disease and death than CRP in non-hospitalized individuals aged 50 to 89 years.

The relationship between NT-pro-BNP levels and long term mortality from all causes in a large cohort of patients with stable coronary heart disease has been assessed by Krageland et $\mathrm{al}^{51}$. NT-pro-BNP was measured in baseline serum samples from 1034 patients referred for angiography. The rate of death from all causes was determined after a median follow-up of nine years. At follow-up, 288 patients had died. The median NT-pro-BNP level was significantly lower among patients who survived than among those who died (120 pg/mL vs.386 $\mathrm{pg} / \mathrm{mL})$.

In a study by Puri et al. the role of NTproBNP in risk stratification and prediction of short-term events including mortality in patients with ACS were evaluated. A total of 120 consecutive patients admitted with a diagnosis of AMI, including both STEMI $(\mathrm{n}=80)$ and NSTEMI $(\mathrm{n}=40)$ were enrolled $^{52}$. In multivariate analysis NTproBNP above median emerged as the strongest predictors of adverse outcomes, including 30-day mortality.

James et al. evaluated whether biomarkers of ischemia, inflammation, myocardial damage, and dysfunction are equally useful in patients who have diabetes mellitus (DM) for prediction of cardiac events in NSTEMI. DM was present in 1,677 of 7,800 patients who had NSTEMI/UA and were included in the Fourth Global Utilization of Strategies ${ }^{53}$. To Open Occluded Arteries (GUSTO IV) trial. Creatinine, NT-proBNP, TnT, CRP, and IL-6 were analyzed in serum samples obtained at a median of 9.5 hours from symptom onset. Oneyear mortality rates were $13.5 \%$ among patients who had DM and $6.9 \%$ among those who did not. The median level of NTpro-BNP was 2 times as high in patients who had DM, whereas troponin $\mathrm{T}$ levels did not differ by DM status.

\section{SUMMARY}

Acute Coronary Syndrome (ACS), i.e., unstable angina or nonST-elevation myocardial infarction (MI) and ST-elevation MI is the most common cause of admission to the coronary care unit with highest risk of death and adverse outcome. ACS accounts for $60-70 \%$ of all admissions in the hospital. Patients with ACS encompass a heterogeneous group that varies widely regarding severity of the underlying coronary artery disease, prognosis and response to treatment. Patients with the highest risk of subsequent events usually have the largest benefit of an intensified pharmacological treatment and early mechanical intervention. The prognosis for low-risk patients, on the other hand, is often difficult to improve further and these patients usually benefit more from a conservative management with a lower risk of side effects. Therefore, risk stratification is essential and should be initiated early and updated continuously throughout the hospital stay.

Early risk stratification is usually performed by the use of clinical background factors, clinical presentation, electrocardiography, and biochemical markers of myocardial damage. Markers of inflammation and renal function have also been shown to be useful. Moreover, measurements of cardiac performance, such as left ventricular ejection fraction and wall motion index, have an important impact on the prognosis. Levels of natriuretic peptides have been shown to reflect cardiac performance. This review focuses on the emerging role of these peptides in the early risk stratification of ACS patients.

In cardiac decompensation, hormones are released from both cardiac and extracardiac origins. Where norepinephrine and endothelin reflect the peripheral responses to cardiac impairment, natriuretic peptides are neurohumoral hormones produced by the heart. Atrial natriuretic peptides (ANP) are mainly expressed in response to atrial myocardial distension, with minute elevations in response to ventricular distension. BNP are natriuretic peptides released by ventricular myocardium, stored mainly in the ventricular myocardium and released into the circulation in response to ventricular dilatation and pressure overload. BNP levels reflect neurohormonal activity and increase with disease progression. They are used currently as prognostic markers in acute coronary syndromes and congestive heart failure.

Elevation of BNP levels in acute MI and UA is predictive of a greater risk of death, postinfarction heart failure, or reinfarction. Postinfarction studies demonstrate that elevated plasma BNP levels are associated with larger infarct size, increased probability of ventricular remodeling, lower ejection fraction, higher risk of heart failure, and increased mortality. This cardiac marker is a potent predictor of mortality in patients with all forms ACS. BNP measurements serve as an index of severity of the ischemic injury, as well as the degree of impairment in left ventricular function. Transient myocardial ischemia, causing an increase in left ventricular wall stress, leads to increased production and release of BNP, even in the absence of necrosis or preexisting left ventricular dysfunction. In homeostatic states, BNP influences natriuresis, inhibits the renin-angiotensinaldosterone system and the sympathetic nervous system activity and facilitates vasodilatation. The release of BNP is thought to be a backup mechanism to ANP, when ANP effects fail to generate adequate cardiac compensation. BNP levels, as a measure of an adverse prognosis, are valid even in the presence of neurohumoral blocking therapy. Levels of BNP during the first day as well as 3 to 4 weeks later following an 
acute coronary event can predict long-term mortality risk and help identify patients risk for adverse outcomes. BNP measurements also help determine the need for aggressive pharmacological and interventional therapies. In acute MI, BNP levels increase rapidly during the first 24 hours and then plateau. Biphsic increase showed additive risk of mortality. In UA with no evidence of necrosis, an increase in BNP levels predicts a worse prognosis. Serial BNP levels provide more prognostic information than a single measurement. The BNP threshold of $80 \mathrm{pg} / \mathrm{mL}$, indicative of neurohormonal activation in heart failure, is similar to that in acute coronary syndromes. Prognostic value of BNP in ACS has been proved as an independent and more significant marker than the other markers of inflammation. It also acts as a risk predictor of mortality and cardiovuscular events in normal population.

\section{CONCLUSION \& RECOMMENDATION}

BNP and NTproBNP are strongly associated to mortality and the risk of future congestive heart failure and carry important prognostic information independent of previously known risk factors in ACS. As such, natriuretic peptides can be added to previously existing risk stratification models and multi-marker approaches. There are some data indicating that these markers might also be helpful in the selection of the appropriate therapy in these patients, but further studies are needed. Before the recommendation of routine measurement of BNP or NTproBNP in ACS, the cost-effectiveness of adding these new markers to the currently used routine markers (e.g. creatinine, troponin and CRP) and their impact on selection of treatment needs further evaluation.

\section{DISCLOSURE}

All the authors declared no competing interest.

\section{REFERENCES}

1. Bayes-Genis A, Mateo J, Santalo M, Oliver A, Guindo J, Badimon L, et al. D-Dimer is an early diagnostic marker of coronary ischaemia in patients with chest pain. Am Heart J 2000; 40: 379-384.

2. Grech ED, Ramsdale DR. Acute coronary syndrome: unstable angina and non ST segment elevation myocardial infarction. British Medical Journal 2003; 326: 1259-1261.

3. Futterman LG, Lemberg L. Novel markers in the acute coronary syndrome: BNP, IL-6, PAPP-A - Cardiology Casebook American Journal of Critical Care 2002; 16:254-261.

4. Puelo PR, Meyer D, Wathen C. Use of a rapid assay of subforms of creatine kinase MB to diagnose or rule out acute myocardial infarction. N Engl J Med 1994; 331: 561-566.

5. ACC/AHA 2002 guideline update for the management of patients with unstable angina and non-ST-segment elevation myocardial infarction. A report of the American College of Cardiology/American Heart Association Task Force on Practice Guidelines (Committee on the Management of Patients with Unstable Angina). Available at :http://www.americanheart.org/presenter.jhtml?identifier=3001260. Accessed May, 2004.

6. James SK, Lindahl B, Siegbahn A, Stridsberg M, Venge P, Armstrong P, et al. N-Terminal Pro-Brain Natriuretic Peptide and Other Risk Markers for the Separate Prediction of Mortality and Subsequent Myocardial Infarction in Patients With Unstable Coronary Artery Disease: A Global Utilization of Strategies To Open occluded arteries (GUSTO)-IV Substudy. Circulation 2003; 108: 275-281.

7. Cheung BMY, Dickerson JEC, Ashby MJ, Brown MJ, Brown J. Effects of physiological increments in human atrial natriuretic peptide and human brain natriuretic peptide in normal male subjects. Clin Sci 1994; 86: 723-730.

8. Maisel A, Krishnaswamy P, Nowak RM, Mc Cord J, Hollander JE, Hermann HC, et al. Rapid measurement of B-type natriuretic peptide in the emergency diagnosis of heart failure. N Engl J Med 2002; 347: 161-167.

9. Omland T, de Lemos JA, Morrow DA, Antman EM, Cannon CP, Hall C, et al. Prognostic value of N-terminal pro-atrial and pro-brain natriuretic peptide in patient with acute coronary syndromes. Am J Cardiol 2002; 89: 463-465.

10. Omland T, Persson A, Ng L, O’Brien R, Karlsson T, Herlitz J, et al. N-terminal Pro-B-Type natriuretic peptide and longterm mortality in acute coronary syndromes. Circulation 2002; 106: 2913-2918.

11. Jernberg T, Stridsberg M, Venge P, Lindahl B. N-terminal pro brain natriuretic peptide on admission for early risk stratification of patients with chest pain and no ST-segment elevation. J Am Coll Cardiol 2002; 40: 437-445.

12. Morrow DA, de Lemos JA, Sabatine MS. Evaluation of B-type natriuretic peptide for risk assessment in unstable angina/non-STelevation myocardial infarction: B-type natriuretic eptide and prognosis in TACTICS-TIMI 18. J Am Coll Cardiol 2003; 41: 1264-1272.

13. Liuzzo G, Biasucci LM, Gallimore JR, Grillo RL, Rebuzzi AG, Pepys MB. The prognostic value of C-reactive protein and serum amyloid a protein in severe unstable angina. N Engl J Med 1994; 331: 417-424.

14. Morrow DA, Rifai N, Antman EM, Weiner DL, McCabe CH, Cannon CP. C-reactive protein is a potent predictor of mortality independently of and in combination with troponin $\mathrm{T}$ in acute coronary syndromes: A TIMI 11A substudy. Thrombolysis in Myocardial Infarction. J Am Coll Cardiol 1998; 31; 1460-1465.

15. Lindahl B, Toss H, Siegbahn A, Venge P, Wallentin L. Markers of myocardial damage and inflammation in relation to long-term mortality in unstable coronary artery disease. Fragmin during Instability in Coronary Artery Disease. N Engl J Med 2000; 343: 1139-1147.

16. De Lemos JA, Morrow DA, Bentley JH, Omland T, Sabatine MS, McCabe CH, et al. The prognostic value of B-type natriuretic peptide in patients with acute coronary syndromes. N Engl J Med 2001; 345: 1014-1021. 


\section{REFERENCES}

17. Sabatine MS, Morrow DA, de Lemos JA, Gibson CM, Murphy SA, Rifai N, et al. Multimarker approach to risk stratification in non-ST elevation acute coronary syndromes: simultaneous assessment of troponin I, C-reactive protein, and B-type natriuretic peptide. Circulation 2002; 105: 1760-1763.

18. Cowie MR, Mendez GF. BNP and congestive heart failure. Prog Cardiovasc Dis 2002; 44: 293-321.

19. Clerico A. Pathophysiological and clinical relevance of circulating levels of cardiac natriuretic hormones: are they merely markers of cardiac disease? Clin Chem Lab Med 2002; 40: 752-760.

20. Kay JD, Trichon BH, Kisslo K, Pentagini M. Serum brain natriuretic peptide levels cannot differentiate pulmonary disease from left-heart failure if the right ventricle is dilated. Circulation 2003; 108 IV: 397.

21. Cowie MR, Jourdain P, Maisel A, Dahistorm U, Follath F, Isnard R, et al. Clinical applications of B-type natriuretic peptide (BNP) testing. Eur Heart J 2003; 24: 1710-1718.

22. Wang TJ, Larson MG, Levy D, Benjamin EJ, Leip EP, Omland T, et al. Plasma natriuretic peptide levels and the risk of cardiovascular events and death. N Engl J Med 2004; 350: 655-663.

23. Nakao K, Ogawa Y, Suga S-I, Imura H. Molecular biology and biochemistry of the natriuretic peptide system. I: Natriuretic peptides. J Hypertens 1992; 10: 907-912.

24. Yandle TG. Biochemistry of natriuretic peptides. J Int Med 1994; 235: 561-576.

25. Sawada Y, Syda M, Yokohama H, Kanda T, Sakamaki T, Tanaka S. Stretch-induced hypertrofic growth of cardiocytes and processing of brain-type natriuretic peptide are controlled by proprotein-processing endoprotease furin. J Biol Biochem 1997; $272: 20545-20554$

26. Hunt PJ, Yandle TG, Nicholls MG, Richards AM, Espiner EA. The amino-terminal portion of pro-brain natriuretic peptide (proBNP) circulates in human plasma. Biochem Biophys Res Comm 1995; 214: 1175-1183.

27. Pemberton CJ, Yandle TG, Rademaker MT, Charles CJ, Aitken GD, Espiner EA. Amino- terminal proBNP in ovine plasma: evidence for enhanced secretion in response to cardiac overload. Am J Physiol 1998 ; 275: 1200-1208.

28. Pemberton CJ, Johnson ML, Yandle TG, Espiner EA. Deconvolution analysis of cardiac natriuretic peptides during acute volume overload. Hypertension 2000; 36: 355-359.

29. Bolger AP, Sharma R, Li W, Leenarts M, Kalra PR, Kemp M. Neurohormonal activation and the chronic heart failure syndrome in adults with congenital heart disease. Circulation 2002; 106: 92-99.

30. Ishi J, Nomura M, Nakamura Y, Naruse H, Mori Y, Ishikawa T, et al. Risk Stratification Using a Combination of Cardiac Troponin T and Brain Natriuretic Peptide in Patients Hospitalized for Worsening Chronic Heart Failure. Am J Cardiol 2002; 89 691-695.

31. Lubien E, DeMaria A, Krishnaswami P, Clopton P, Koon J, Kazanegra R, et al. Utility of B- Natriuteric Peptide in Detecting Diastolic Dysfunction: Comparison with doppler velocity recordings. Circulation 2002; 105: 595-601.

32. Coletta A, Thackray S, Nikitin N, Cleland JG. Clinical trials update: highlights of the scientific sessions of The American College of Cardiology 2002: LIFE, DANAMI 2, MADIT-2, MIRACLE-ICD, OVERTURE, OCTAVE, ENABLE 1 \& 2, CHRISTMAS, AFFIRM, RACE, WIZARD, AZACS, REMATCH, BNP trial and HARDBALL. Eur J Heart Fail 2002; 4: 381 388.

33. De Lemos JA, Morrow DA. Brain natriuretic peptide measurement in acute coronary syndromes: ready for clinical application? Circulation 2002; 106: 2826-2870

34. De Lemos JA, Morrow DA, Gibson CM, Murphy SA, Sabatine MS, Rifai N, et al. The prognostic value of serum myoglobin in patients with non-ST-segment elevation acute coronary syndromes Results from the TIMI 11B and TACTICS-TIMI 18 studies. J Am Coll Cardiol 2002; 40: 238-244.

35. Vasan RS, Benjamin EJ, Larson MG, Leip EP, Wang TJ, Wilson PW. Plasma natriuretic peptides for community screening for left ventricular hypertrophy and systolic dysfunction: the Framingham heart study. JAMA 2002; 288: 1252-1259.

36. Hunt PJ, Espiner EA, Nicholls MG, Richards AM, Yandle TG. The role of the circulation in processing pro-brain natriuretic peptide (proBNP) to amino-terminal BNP and BNP-32. Peptides 1997; 18: 1475-1481.

37 Talwar S, Squire IB, Downie PF, Davies JE, Ng LL. Plasma N terminal pro-brain natriuretic peptide and cardiotrophin 1 are raised in unstable angina. Heart 2000; 84: 421-424.

38. Arakawa N, Nakamura M, Aoki H, Hiramori K. Relationship between plasma brain natriuretic peptide and myocardial infarct size. Cardiology 1994; 85: 334-340.

39. Arakawa N, Nakamura M, Aoki H, Hiramori K. Plasma brain natriuretic peptide concentrations predict survival after myocardial infarction. J Am Coll Cardiol 1996; 27: 1656-1661.

40. Omland T, Aakvaag A, Bonarjee VV, Caidahl K, Lie RT, Nilsen DW, et al. Plasma brain natriuretic peptide as an indicator of left ventricular systolic function and long-term survival after acute myocardial infarction. Comparison with plasma atrial natriuretic peptide and N-terminal proatrial natriuretic peptide. Circulation 1996; 93: 1963-1969.

41. Saribulbul O, Alat I, Coskun S, Apaydin AZ, Yagdi T, Kiliccioglu M, et al. The Role of Brain Natriuretic Peptide in the Prediction of Cardiac Performance in Coronary Artery Bypass Grafting. Tex Heart Inst J 2003; 30: 298-304. 


\section{REFERENCES}

42. Jernberg T, James S, Lindahl B, Johnston N, Stridsberg M, Venge P, et al. Natriuretic peptide in unstable coronary artery disease. Euro H Journal 2004; 25: 1486-1493.

43. Bazzino O, Fusseli JJ, Botto F, Arenaza DP, Bahit C, Dadone J. Relative value of probrain natriuretic peptide, TIMI risk score, ACC/AHA prognostic classification and other risk marker in patients with non ST elevation acute coronary syndrome. Euro $\mathrm{H}$ Journal 2004; 25: 859-866.

44. Yang JC, Nan L, Jian W. Use of B-type natriuretic peptide in evaluation of early percutaneous coronary intervention in patients with acute coronary syndrome Chin Med J 2004; 117: 1130- 1134.

45. Galvani M, Ottani F, Oltrona L, Ardissino D, Gensini GF, Maggioni AP, et al. N-Terminal Pro-Brain Natriuretic Peptide on Admission has Prognostic Value Across the Whole Spectrum of Acute Coronary Syndromes. Circulation 2004; 110: 128-134.

46. Ollivier JP, Revel F. Brain natriuretic peptide in acute coronary syndromes: utility of NT-pro BNP assay. Bull Acad Natl Med 2004; 188:1529-1532.

47. Suzuki S, Yoshimura M, Nakayama M, Mizuno Y, Harada E, Ito T, et al. Plasma Level of B-Type Natriuretic Peptide as a Prognostic Marker After Acute Myocardial Infarction: A Long-Term Follow-Up Analysis. Circulation 2004; 110: 1387-1391.

48. Jarai R, Iordanova N, Jarai R, Wolfgang AR. Risk assessment in patients with unstable angina/non-ST-elevation myocardial infarction and normal N-terminal pro-brain natriuretic peptide levels by N-terminal pro-atrial natriuretic peptide. Rev Esp Cardiol 2004; 56: 389-395

49. Heeschen C, Hamm CW, Mitrovic V, Lantelme NH, White HD. Platelet Receptor Inhibition in Ischemic Syndrome Management (PRISM) Investigators. N-terminal pro-B-type natriuretic peptide levels for dynamic risk stratification of patients with acute coronary syndromes. Circulation 2004; 110: 3206-3212.

50. Kistorp C, Raymond I, Pedersen F, Gustafsson F, Faber J, Hildebrandt P. N-Terminal Pro-Brain Natriuretic Peptide, C-Reactive Protein, and Urinary Albumin Levels as Predictors of Mortality and Cardiovascular Events in Older Adults. JAMA 2005; 293: 1609-1616.

51. Kragelund C, Grønning B, Køber L, Hildebrandt P, Steffensen R. N-Terminal Pro-B-Type Natriuretic Peptide and Long-Term Mortality in Stable Coronary Heart Disease. N Engl J Med 2005; 352: 666-675.

52. Puri A, Narain VS, Mehrotra S, Dwivedi SK, Saran RK, Puri VK. N-terminal probrain natriuretic peptide as a predictor of shortterm outcomes in acute myocardial infarction. Indian Heart J 2005; 57: 304-310.

53. James SK, Lindahl B, Timmer JR, Ottervanger JP, Siegbahn A, Stridsberg M, Usefulness of biomarkers for predicting longterm mortality in patients with diabetes mellitus and non-ST-elevation acute coronary syndromes (a GUSTO IV substudy). Am J Cardiol 2006; 97: 167-172. 